\title{
Research Trends and Correlation Analysis of Technological Innovation and Absorptive Capacity
}

\author{
Eun-Mi Park ${ }^{1}$, Joung-Hae Seo ${ }^{2 *}$ \\ ${ }^{1,2}$ Department of Business Administration, Kyungpook National University, Daegu, 41566, South Korea \\ *Corresponding author: E-mail: knight6407@naver.com
}

Article History: Received:11 november 2020; Accepted: 27 December 2020; Published online: 05 April 2021 \begin{tabular}{l} 
Abstract: The purpose of this study is to identify the research trend of innovation capacity and technological innovation that \\
are recently required in companies. This was done by collecting and analyzing dissertation abstracts from Springer and \\
Scopus, which are websites with a collection of dissertations. For the research method, this study used NetMiner 4 and R \\
program for text data analysis amongst unstructured data analysis, and analysis methods like keyword network analysis, \\
LDA, and TreeMap were applied. The analysis results showed that there is a difference in the importance of technological \\
innovation and absorptive capacity of Springer and Scopus \\
Keywords: Technological Innovation, Absorptive Capacity, LDA, Network Analysis, TF-IDF \\
\hline
\end{tabular}

\section{Introduction}

Unlike the past, many changes are happening in our lives because of rapid technological advancement[1]. Recently, we have entered the Fourth Industrial Revolution followed by the First Industrial Revolution, Second Industrial Revolution, and Third Industrial Revolution. Various technologies are included in the Fourth Industrial Revolution. It was possible through innovation like IoT, big data, artificial intelligence, and Cloud[2,3]. Many developed countries, along with global companies, are competing on a national level to establish and support various policies for technological innovation. In particular, they are providing all their capacity to secure source technology. Currently, Singapore ranks 1st in the world competitive ranking of the Fourth Industrial Revolution followed by United States as 2nd, Hong Kong as 3rd, and South Korea coming in at 13th. Although the ranking has elevated significantly compared to the previous year, much more efforts are still required. In the Fourth Industrial Revolution referred to as ICBMA[1,2], technological innovation that corresponds should proceed in order for corporations to survive.

In other words, it is different from the past, as efforts are required in order to be equipped with the capacity to develop and apply the newest technology. Recently, many companies are racing to do their best for technological innovation, but because they are only focused on developing technology, they do not make an investment for the measures to apply the technology properly in the companies. In addition, in case the companies lack in their capacity of technological development, then externally developed technology has to be applied. Accordingly, absorptive capacity is required to apply the outside technology and knowledge internally[4]. In this study, the research trend of technological innovation and absorptive capacity trends are to be identified. Especially, the relevance between technological innovation and absorptive capacity will be identified and NetMiner will be applied for network analysis technique to execute the analysis. Moreover, technological innovation and absorptive capacity were the only dissertation titles searched on Springer and Scopus dissertation database.

This study will follow the following procedures. First, the introduction will explain the background and purpose of this study. In section 2, the theoretical background will be discussed. In section 3, the data analysis procedures and method will be described, followed by the research findings in section 4 and the conclusion and implication in section 5 .

\section{Related Work}

\subsection{Technology Innovation}

Technology is a complicated field with the properties of rapid changes. In addition, companies are continuously making an attempt to create new markets, reduce the production cost, and bring differentiation by using technology as a measure to secure the competitive advantage. Schumpeter(1934) defined technological innovation as creative destruction and the innovation stages were classified into new production development stage, new production method adaptation and new technique development stage, new market colonization stage, new supply stage of raw materials, and new organization formation stage[5].

In addition, Schmookler(1966) based on the existing Schumpeter research and classified technological innovation into products and process innovation[6]. Damanpour \& Evan(1984) claimed that newly applying technological innovation onto new products, service, system, procedures, program, and device to an organization through technological innovation and promoting changes in the organization represents technological innovation[7]. In the research by Park et al.(2018), technological innovation was categorized into product innovation, process innovation, marketing innovation, organization innovation, innovation that

*Corresponding author: Joung-Hae Seo 
reinforces key capabilities, innovation that destroys the key capabilities, product structure innovation, and parts innovation[8].

\subsection{Absorptive Capacity}

Absorptive capacity is the ability of an organization to convert and apply to acquire and digest outside knowledge and apply it for the intention[9,10]. In addition, the important fact is that absorptive capacity is linked to innovation and regarded as the key source of successful innovation[11,12]. While the absorptive capacity of Cohen \& Levinthal(1990) was analyzed as the capacity of the company to absorb knowledge from the affiliated field[10], the absorptive capacity of Lane \& Lubatkin(1998) was analyzed as the capacity to absorb knowledge from other companies and defined as applying and understanding the knowledge that results from other companies[13]. Zahra \& George(2002) based on the concept of absorptive capacity of Cohen \& Levinthal(1990) and claimed that the four competence including the acquisition, digestion, conversion, and application of external knowledge cause a path-dependency influence[14].

\subsection{Text mining}

The purpose of text mining is to analyze the text data to identify the rules and the patterns and derive useful information. Generally, text mining collects data and executes the process of converting with noise removal, tokenization, POS tagging, stopword removal, and vector expression and applying the algorithm. By using text mining technology, natural language processing technique is applied first in the human centered unstructured data to extract meaningful number or word index from the extracted text[15]. The information included in the text is made so that it can be approached through various text mining algorithm like statistics or regular machine learning to extract meaningful information. In addition, text mining is the technique that is the basis of other unstructured data mining techniques like web mining, opinion mining, or social network analysis.

\subsection{Network Analysis}

Keyword network makes it possible to connect the keyword from the text and also extract the network of those concepts[16]. Generally, network analysis method includes network cohesion analysis, sub-network analysis, structural equivalence analysis, and centrality analysis. Additionally, measurement indicators to analyze the network cohesion include inclusiveness, degree, density, and clustering coefficient[17]. Keyword network analysis makes it possible to conduct structural analysis additionally through clustering analysis and centrality analysis, in addition to simple frequency analysis, and it has the advantage where methods for qualitative or quantitative analysis can be applied at the same time. In addition, the measurement indicators of centrality are degree centrality, betweenness centrality, and closeness centrality.

\subsection{Latent Dirichlet Allocation}

In natural language processing, Latent Dirichlet Allocation(LDA) refers to a probability model regarding topics existing in each given document[18,19]. Document topic distribution value $(\theta)$, word distribution value $(\beta)$ of a topic and the number of topics $(\mathrm{k})$ should be entered as hyper parameters beforehand. Based on the values entered, words (w) were observed, and each word was given an appropriate topic number (z).

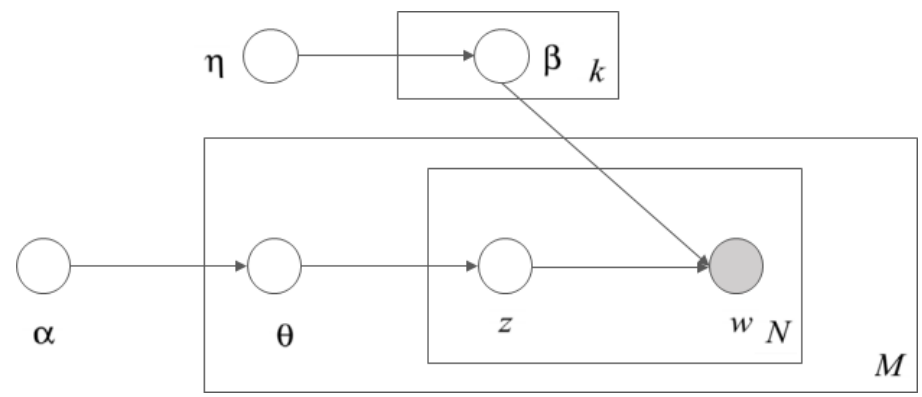

- M: Number of documents

- N: Number of words in the document

- W: word

- Z: Topic number to which the word belongs

- $\mathrm{k}$ : Number of topics (Hyper parameter)

- $a$ : Document-topic $\theta$ distribution value (Hyper parameter) 
- $n$ : Topic-word-by-word $\beta$ distribution value (Hyper parameter)

- $\theta$ : Distribution of topics by document

- $\beta$ : Word distribution of topics

\section{Research Procedure}

This study collected data with the following measures and conducted an analysis. First, Springer database was applied in NetMiner and abstracts were downloaded from the dissertation database of Scopus. For the collected data, technological innovation appeared as 103 for Springer and 701 for Scopus. In case of the absorptive capacity, Springer had 87 and Scopus had 92. On Springer, the dissertation title was limited through NetMiner and the keyword was set up as 'technological innovation' and 'absorptive capacity' to execute the analysis. On Scopus, the website was accessed and it was limited first as the dissertation title and the keyword were set up as 'technological innovation' and 'absorptive capacity,' the document type was set up as articles, and the keyword was fixed as technological innovation to conduct the analysis,

The collected data was set up so that it is suitable for network analysis through data cleansing and data pre-processing. Then, NetMiner and $\mathrm{R}$ were used to implement the keyword network, LDA, and TreeMap analysis. The data collection method and keyword network analysis method and procedures through NetMiner are as shown in figure 3. The methods and procedures of LDA and TreeMap analysis through R are as shown in figure 4.

Figure 1. Number of papers published in technological innovation and absorption capacity(scopus)

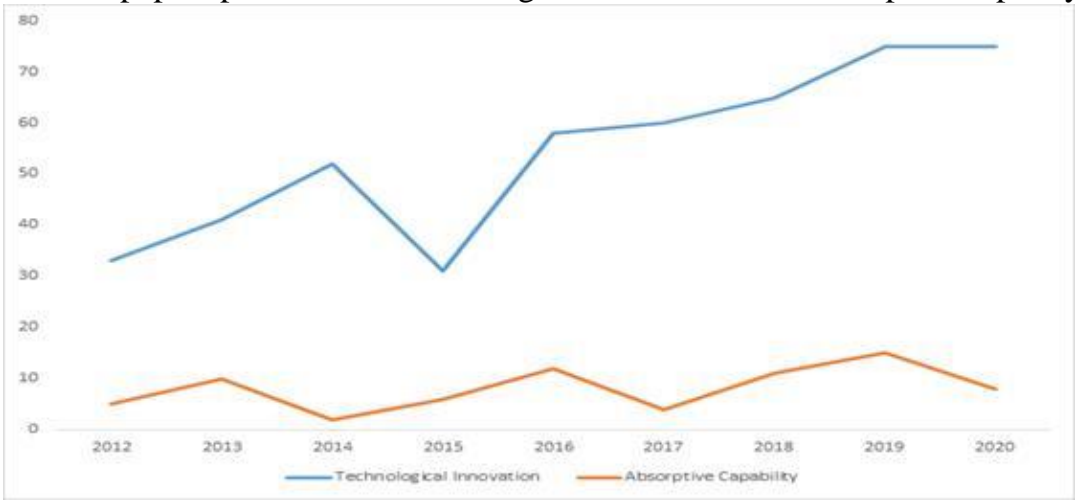

\section{Results}

\subsection{Keyword Network (Springer)}

\subsubsection{Technological Innovation Analysis Results}

Weight refers to the link frequency of the created word pair. In this study, 7 times and above was chosen as the standard. The \# of sentences signifies the number of sentences/paragraphs/documents where the particular word pair appeared. First of all, it was displayed that innovation and performance are important. Weight appeared 20 times and the \# of sentences as 11 and the Gini coefficient value appeared as 0.9. The next importance were the capacity and the knowledge. Weight appeared 28 times and the \# of sentences appeared 19 times, and the Gini coefficient value appeared as 0.9. Aside from this, it was displayed that the capacity and innovation, knowledge and transfer are also important was shown in the Table 1.

Table 1. Keyword Network Analysis Results (Technological Innovation)

\begin{tabular}{|c|c|c|c|c|}
\hline Source & Target & Weight & \# of Articles & Gini Coefficient \\
\hline capacity & development & 7 & 7 & 0.9 \\
\hline capacity & capability & 7 & 6 & 0.9 \\
\hline UIC & innovation & 7 & 1 & 1 \\
\hline firm & capacity & 8 & 7 & 1 \\
\hline supply & chain & 8 & 2 & 1 \\
\hline RnD & cooperation & 14 & 2 & 1 \\
\hline training & performance & 8 & 3 & 1 \\
\hline productivity & growth & 8 & 2 & 0.9 \\
\hline innovation & capability & 8 & 8 & 0.9 \\
\hline capacity & firm & 8 & 8 & 1 \\
\hline role & capacity & 8 & 3 & 0.9 \\
\hline innovation & network & 8 & 19 & \\
\hline capacity & knowledge & 28 & & \\
\hline
\end{tabular}




\begin{tabular}{|c|c|c|c|c|}
\hline knowledge & spillover & 15 & 4 & 1 \\
\hline concept & capacity & 7 & 7 & 0.9 \\
\hline business & performance & 10 & 3 & 0.9 \\
\hline capacity & performance & 7 & 6 & 0.9 \\
\hline capacity & study & 7 & 5 & 0.9 \\
\hline knowledge & capacity & 13 & 6 & 0.9 \\
\hline level & capacity & 7 & 11 & 0.9 \\
\hline innovation & performance & 30 & 15 & 0.9 \\
\hline capacity & innovation & 23 & 6 & 0.9 \\
\hline transformation & exploitation & 7 & 8 & 0.9 \\
\hline knowledge & transfer & 20 & 7 & 1 \\
\hline performance & result & 7 & 5 & 0.9 \\
\hline subsidiary & capacity & 8 & 7 & 1 \\
\hline manufacturing & firm & 9 & 2 & 1 \\
\hline science & technology & 7 & 4 & \\
\hline effect & relationship & 7 & & \\
\hline
\end{tabular}

In other words, technological innovation is closely linked to performance, knowledge, and capacity. This is the keyword network results based on the entire data where the analysis was conducted was shown in the Figure 2.
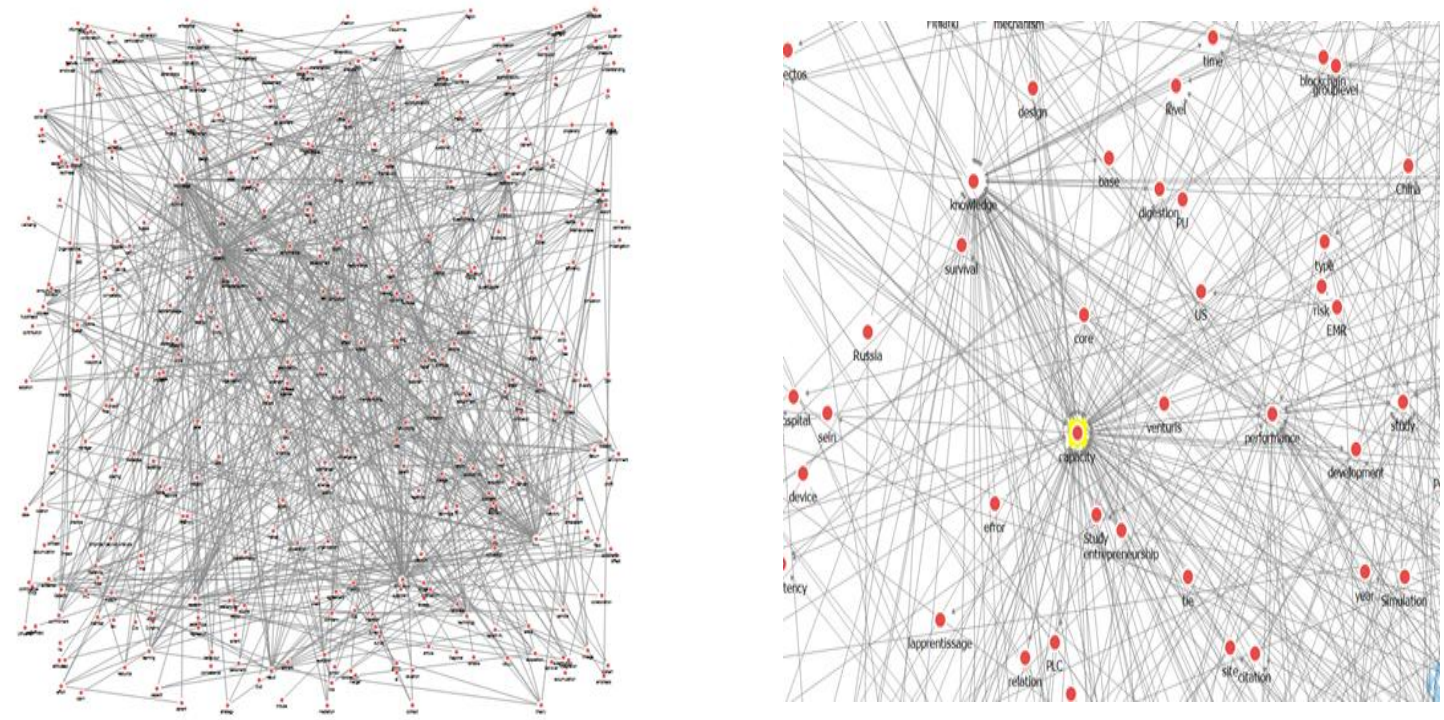

Figure 2. Visualize network analysis results (Technological Innovation)

When this is examined closely, the important terms are derived as follows. The important terms were capacity, performance, and knowledge. TF-IDF weighting method was used to deduce important terms, The TFIDF weight can be displayed like the following formula. The weight $\mathrm{w}_{i, j}$ of keyword $i$ that is affiliated to document $j$ is the product of $\mathrm{tf}$ value and df value[20].

$$
\begin{aligned}
t f i d f(\mathrm{t}, \mathrm{d}, \mathrm{D}) & =t f(t, d) \times \operatorname{idf}(\mathrm{t}, \mathrm{D}) \\
i d f(\mathrm{~d}, \mathrm{t}) & =\log \frac{n}{1+d f(t)}
\end{aligned}
$$

Here, $\mathrm{tf} i, j$ is the frequency of keyword $i$ that appeared in document $j$, and $\mathrm{df} i, j$ is the value that is gained by taking the $\log$ from the inverse number of the number of document containing keyword $i$. The following are the results of executing word-article net(TD_IDF). The results of only selecting the weight that is 1.2 and above out of the overall data is as shown in the following table 2.

Table 2. TF-IDF analysis result (Technological Innovation)

\begin{tabular}{|c|c|c|c|}
\hline Source & Weight & Source & Weight \\
\hline inflow & 1.2 & accumulation & 1.2 \\
\hline inventor & 1.4 & memory & 1.4 \\
\hline district & 1.3 & risk & 1.4 \\
\hline
\end{tabular}




\begin{tabular}{|c|c|c|c|}
\hline valuation & 1.2 & networking & 1.2 \\
\hline announcement & 1.2 & competence & 1.4 \\
\hline internacionales & 1.2 & ICT & 1.2 \\
\hline improvisation & 1.2 & intention & 1.2 \\
\hline member & 1.2 & GBS & 1.2 \\
\hline PLC & 1.4 & savoirs & 1.3 \\
\hline Vietnam & 1.2 & site & 1.2 \\
\hline delivery & 1.2 & office & 1.2 \\
\hline subsidy & 1.4 & training & 1.3 \\
\hline team & 1.5 & science & 1.2 \\
\hline attitude & 1.2 & lapprentissage & 1.3 \\
\hline startups & 1.2 & emission & 1.4 \\
\hline survival & 1.2 & dveloppement & 1.2 \\
\hline EMR & 1.4 & wellbeing & 1.2 \\
\hline supply & 1.5 & continuance & 1.2 \\
\hline filter & 1.4 & trait & 1.3 \\
\hline selfefficacy & 1.2 & aprendizaje & 1.2 \\
\hline buyersupplier & 1.2 & PAC & 1.3 \\
\hline NPD & 1.3 & Lithuania & 1.2 \\
\hline organisation & 1.3 & assignment & 1.2 \\
\hline entre & 1.3 & blockchain & 1.2 \\
\hline
\end{tabular}

The terms that had high weight were supply, team, PLC, and NPD(new product development). This means that it is important to apply it for supply through technological innovation, team formation for technological innovation development, product life cycle, and new product development.

\subsubsection{Absorptive Capacity Analysis Results (Springer)}

The following are the absorptive capacity analysis results. Weight refers to the link frequency of the created word pair. In this study, 7 times and above was chosen as the standard. The \# of sentences signifies the number of sentences/paragraphs/documents where the particular word pair appeared. First of all, it was displayed that innovation and performance are important. Weight appeared 48 times and the \# of sentences as 47 and the Gini coefficient value appeared as 0.5 . The next importance were the capacity and the knowledge. Weight appeared 48 times and the \# of sentences appeared 9 times, and the Gini coefficient value appeared as 0.9. Aside from this, it was displayed that the capacity and innovation, knowledge and transfer are also important was shown in the Table 3.

Table 3. Keyword Network Analysis Results (Absorptive Capacity)

\begin{tabular}{|c|c|c|c|c|}
\hline Source & Target & Weight & \# of Articles & Gini Coefficient \\
\hline innovation & enterprise & 14 & 8 & 0.9 \\
\hline Total factor & energy & 7 & 1 & 1 \\
\hline supply & chain & 9 & 3 & 1 \\
\hline energy & efficiency & 9 & 1 & 0.9 \\
\hline effect & innovation & 7 & 7 & 1 \\
\hline risk & measure & 7 & 2 & 1 \\
\hline innovation & strategy & 8 & 3 & 1 \\
\hline efficiency & enterprise & 9 & 2 & \\
\hline
\end{tabular}




\begin{tabular}{|c|c|c|c|c|}
\hline enterprise & innovation & 13 & 8 & 0.9 \\
\hline innovation & capability & 24 & 9 & 0.9 \\
\hline impact & innovation & 8 & 7 & 0.9 \\
\hline innovation & system & 12 & 7 & 1 \\
\hline equipment & industry & 9 & 3 & 1 \\
\hline development & innovation & 7 & 7 & 0.9 \\
\hline adoption & innovation & 9 & 5 & 1 \\
\hline innovation & process & 13 & 9 & 0.9 \\
\hline science & technology & 7 & 4 & 1 \\
\hline innovation & efficiency & 19 & 6 & 1 \\
\hline technology & innovation & 19 & 14 & 0.9 \\
\hline innovation & development & 9 & 4 & 1 \\
\hline Technological & innovation & 10 & 10 & 0.9 \\
\hline Total factor & efficiency & 7 & 1 & 1 \\
\hline investment & innovation & 7 & 5 & 1 \\
\hline innovation & paper & 10 & 10 & 0.9 \\
\hline innovation & management & 9 & 3 & 1 \\
\hline performance & bank & 8 & 1 & 1 \\
\hline Technological & Innovation & 48 & 47 & 0.5 \\
\hline Technological & Innovations & 10 & 10 & 0.9 \\
\hline & & & & \\
\hline & & & 10 & \\
\hline & & 9 & 10 \\
\hline
\end{tabular}

In other words, absorptive capacity is closely linked to technological innovation. This is the keyword network results based on the entire data where the analysis was conducted was shown in the Figure 3.
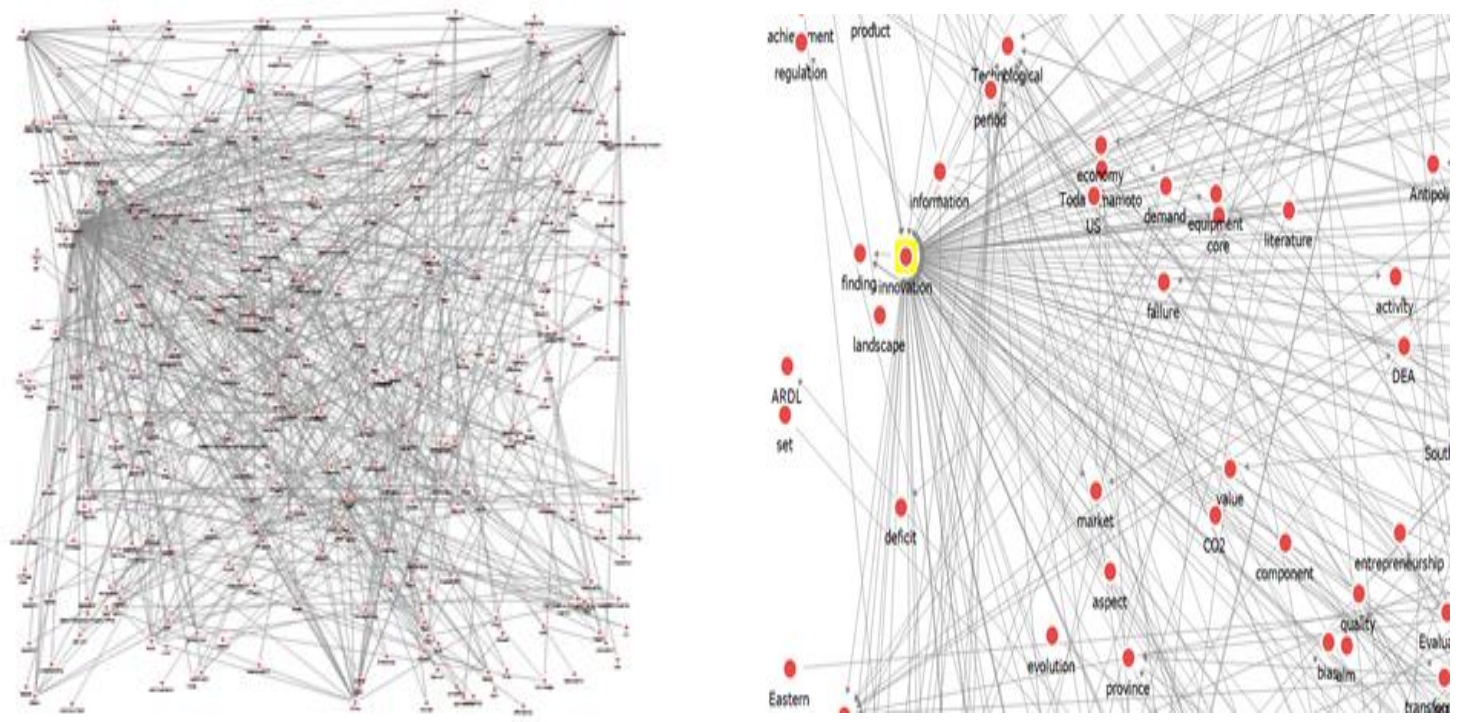

Figure 3. Visualize network analysis results(Absorptive Capacity)

To sum up, it was displayed that there is a rather close connection between absorptive capacity and technical innovation, and innovation. TF-IDF weighting method was used as the method to derive important terms. TF-IDF weight can be displayed like the following formula.

The value of keyword $i$ that is affiliated to document $j$ is the product of tf value and df value. Here, $\operatorname{tf}_{i, j}$ is the frequency of keyword $i$ that appeared in document $j$, and $\mathrm{d} \mathrm{tf}_{i, j}$ is the value of taking the log from the inverse number of the number of document containing keyword $i$. The following is the outcome of executing wordarticle net(TF-IDF). The results of only selecting the weight that is 1.2 and above out of the overall data is as 
shown in the following table 4.

Table 4. TF-IDF analysis result (Absorptive Capacity)

\begin{tabular}{|c|c|c|c|}
\hline Source & Weight & Source & Weight \\
\hline bank & 1.4 & BRICS & 1.3 \\
\hline Total factor & 1.4 & Sophia & 1.3 \\
\hline family & 1.3 & Antipolis & 1.3 \\
\hline PV & 1.3 & PLC & 1.4 \\
\hline transaction & 1.4 & distortion & 1.3 \\
\hline Anhui & 1.4 & leadership & 1.3 \\
\hline affordance & 1.3 & engagement & 1.3 \\
\hline coal & 1.4 & & \\
\hline
\end{tabular}

The terms with high weight were displayed as PLC, coal, transaction, and bank. It appeared that product life cycle, coal, and bank are important factors for absorptive capacity. This is the keyword network results that base on the entire data where the analysis was performed. When it was examined closely, the important terms were displayed as innovation and technological.

\subsection{LDA}

The key words include innovation, air, model, mechanism, and technological, from which it can be inferred that 'innovation' is the topic. The verb key words include such positive key words. The lower the gamma value, the longer the red bar(Figure.4).

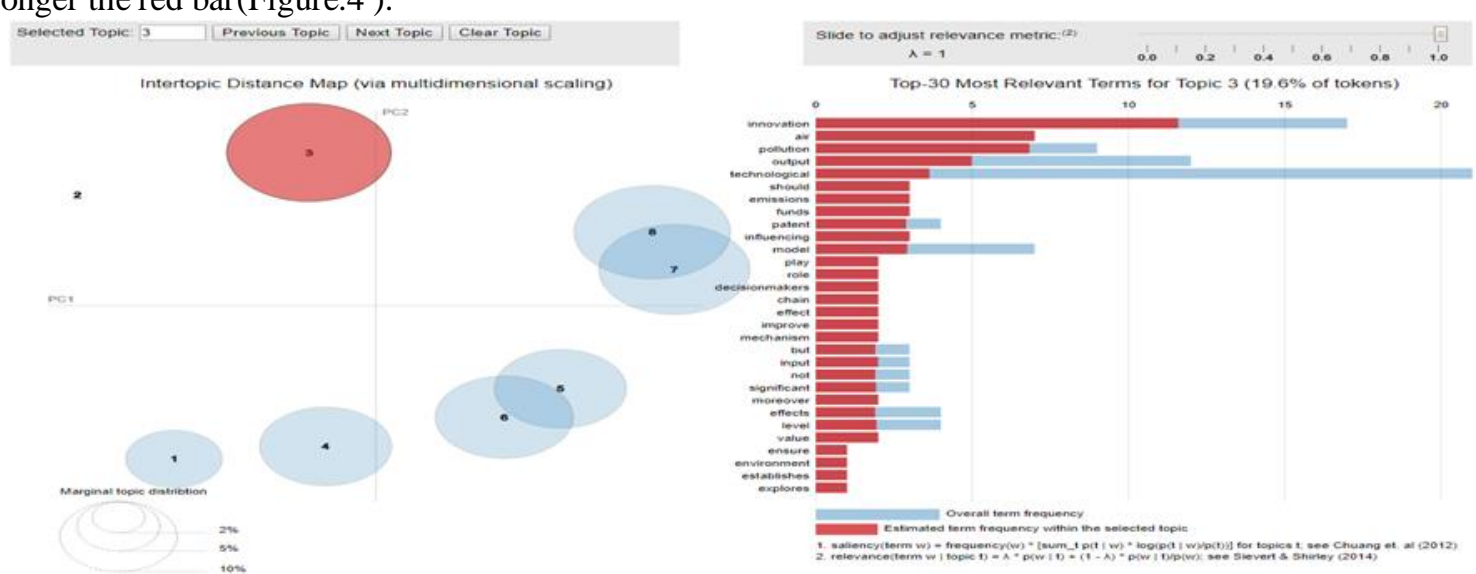

Figure 4. LDA analysis result 1 (Technological Innovation)

No.7 and No.8 topics are similar to each other. The key words include technological, scientific, potential, opportunity, and advancement, from which it can be inferred that 'technology' is the topic. The verb key words include such positive key words. The lower the gamma value, the longer the red bar(Figure. 5).

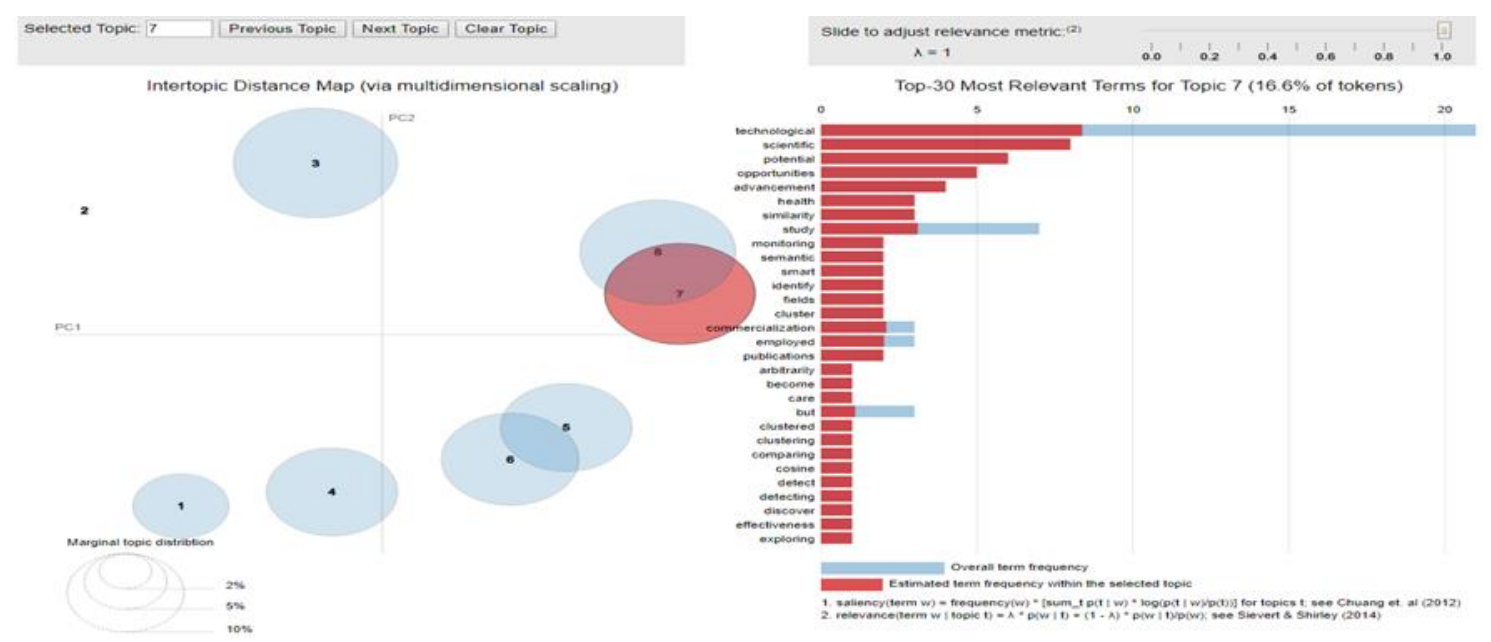

Figure 5. LDA analysis result 2 (Technological Innovation) 
No.3 and No.5 topics are similar to each other. The key words include absorptive, capabilities, performance, innovation, and knowledge, from which it can be inferred that 'absorptive' is the topic. The verb key words include such positive key words. The lower the gamma value, the longer the red bar (Figure.6 ).
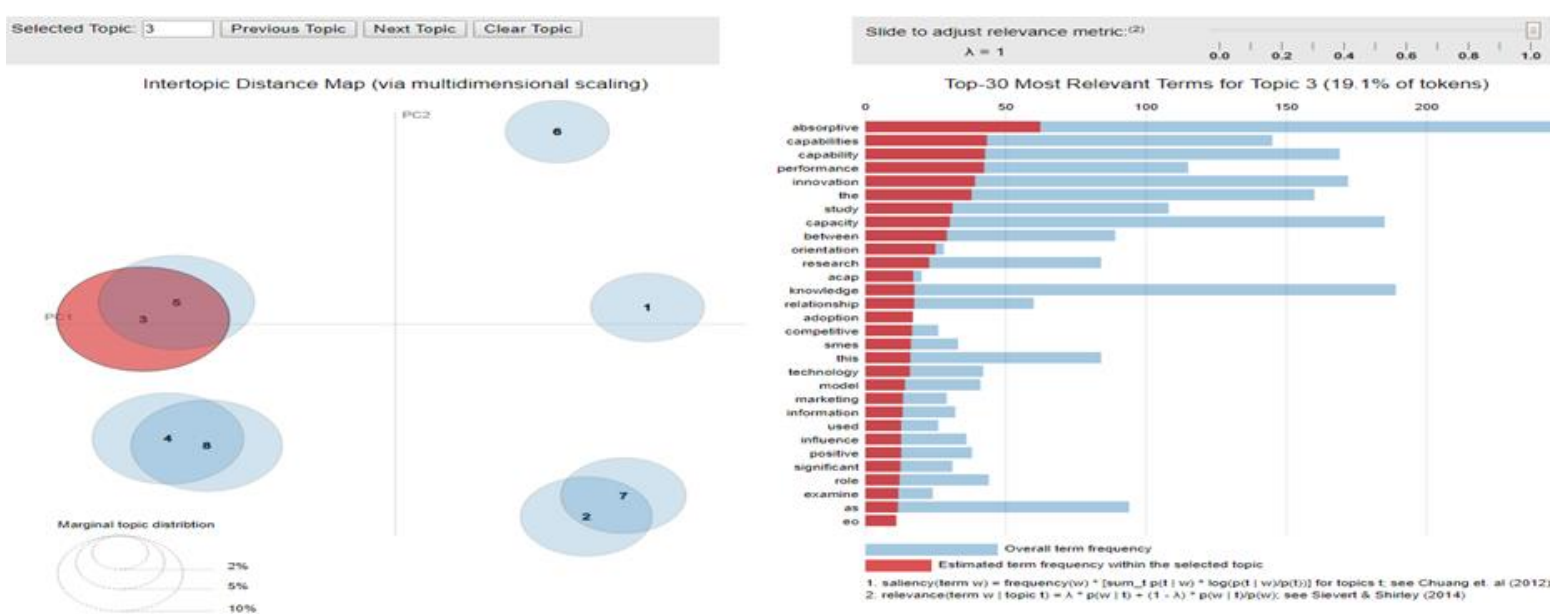

Figure 6. LDA analysis result 1(Absorptive Capacity)

No.4 and No.8 topics are similar to each other. The key words include absorptive, capability, knowledge, performance, innovation, and relationship, from which it can be inferred that 'absorptive' is the topic. The verb key words include such positive key words. The lower the gamma value, the longer the red bar (Figure. 7).
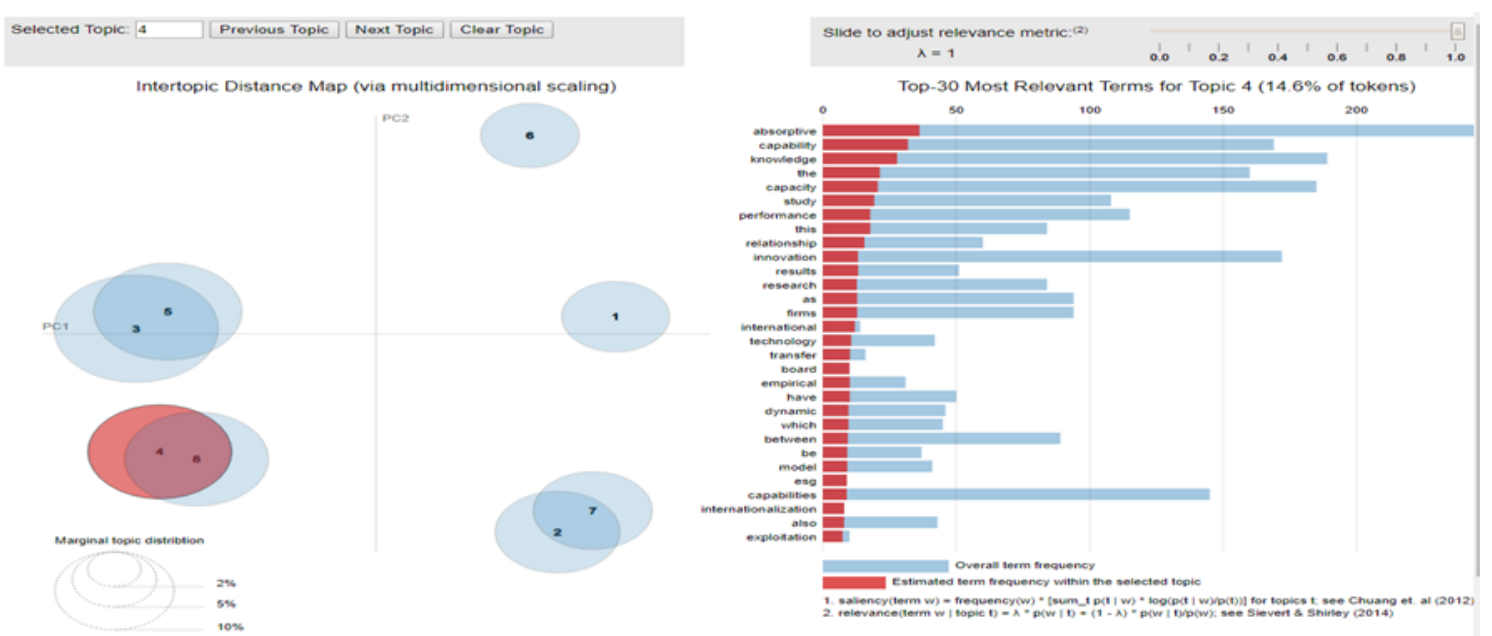

Figure 7. LDA analysis result 2(Absorptive Capacity)

\subsection{Technology Innovation}

The following are the results when displayed with Word Cloud. Adoption, calves, and transfer appeared as important factors. This means that big size is important in Treemap. The important terms were adoption, transfer, vitamin, competences, expert, and innovativeness was shown Figure 8. 

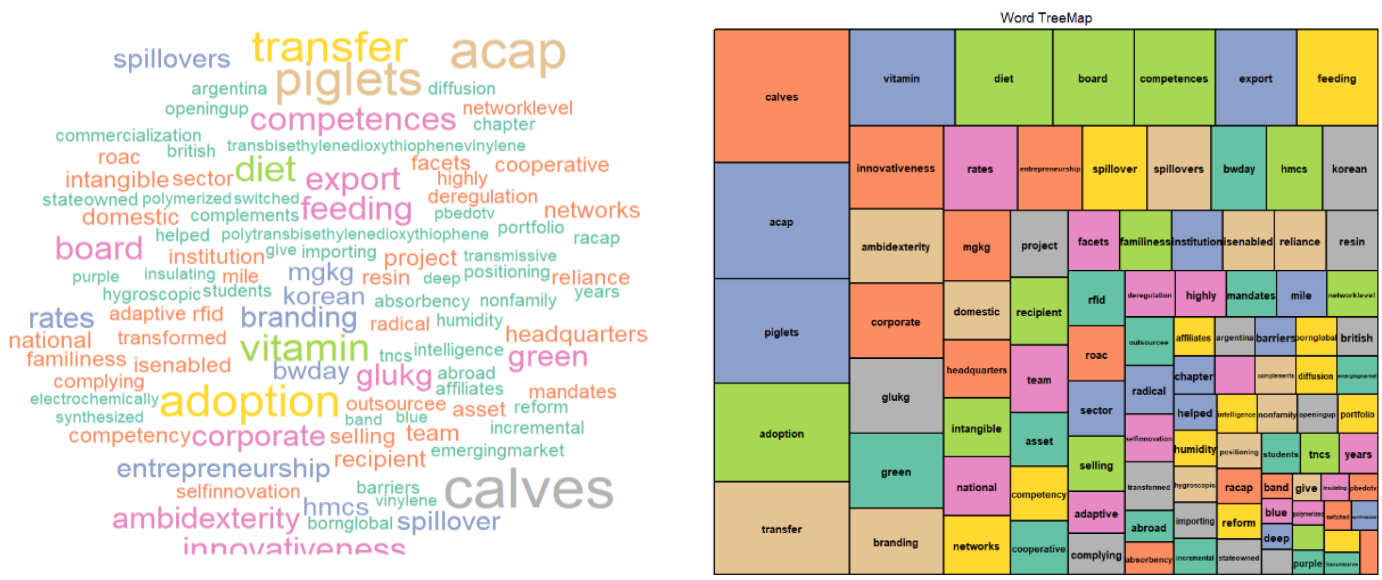

Figure 8. Wordcloud and TreeMap

\subsection{Discussion}

In this study, abstracts of 'technological innovation' and 'absorptive capacity' were collected from Springer and Scopus database and an analysis was performed. First of all, there were differences in the terms derived as important terms in the data analysis collected from Springer through NetMiner 4 and the terms derived as important from the data analysis collected from Scopus database. It can also be identified through the differences of significance between Springer and Scopus, but it seems like it is due to the differences in the amount of the data. However, in common, technological innovation and absorptive capacity are closely connected. In order for technological innovation to be successful, absorptive capacity is certainly required. From the company's position, research has to be performed in various ways to heighten the absorptive capacity instead of only focusing on technological innovation. Moreover, future studies will more likely to focus on further detailed causality instead of the simple causality of technological innovation and absorptive capacity in accordance with the recent trend.

\section{Conclusions}

The research findings of this study are as follows. The following are the important findings of technological innovation. innovation and performance, capacity, and knowledge appeared to be important. importance on innovation and technological, scientific, and potential. The following are the important findings of absorptive capacity. technological and innovation, innovation and capability appeared to be important. absorptive and capability, performance, and knowledge appeared to be important. In digital transformation times, technology and innovation represented by DNA are most important, so researches about it are likely to increase. In particular, absorptive capacity is important for technological innovation to be successful, more than the influential factor caused by technological innovation on the performance, so convergence research about it is likely to increase.

From the company's perspective, various measures are required to survive in the fierce competition. In the Convergence Age, it seems more appropriate to perform convergence research through the integral of different fields rather than only doing research on a single field. Since this study suggested the research trend and research directivity through abstracts of dissertation about technological innovation and absorptive capacity in academic database, it provides an academic implication at an academic level. In addition, the corporate situation about convergence research comes from practical implication. However, the limitation of this study is that other dissertation database were not taken into consideration besides the 2 dissertation database. Accordingly, various dissertation database data needs to be used in future research, and it is necessary to apply various big data analysis techniques.

\section{References}

1. Park, Seong-Taek, Do-Yeon Kim, and Guozhong Li. "An analysis of environmental big data through the establishment of emotional classification system model based on machine learning: focus on multimedia contents for portal applications." Multimedia Tools and Applications (2020): 1-19.

2. Park, Seong-Taek, Jae-Rim Jung, and Chang Liu. "A study on policy measure for knowledge-based management in ICT companies: focused on appropriability mechanisms." Information Technology and Management 21, no. 1 (2020): 1-13.

3. Park, Seong-Taek, Guozhong Li, and Jae-Chang Hong. "A study on smart factory-based ambient intelligence context-aware intrusion detection system using machine learning." Journal of Ambient Intelligence and Humanized Computing 11, no. 4 (2020): 1405-1412. 
4. Park, Eun-Mi, and Joung-Hae Seo. "A study on effects of authentic leadership on job stress, SelfEfficacy, organizational effectiveness." Indian Journal of Public Health Research \& Development 9, no. 8 (2018): 380-386.

5. Schumpeter, Joseph A. "The theory of economic development: An inquiry into profits, capital, credit, interest, and the business cycle (1912/1934)." Transaction Publishers.-1982.-January 1 (1982): 244.

6. Schmookler, Jacob. "Invention and Economic Growth (Cambridge, Mass., 1966)." SchmooklerInvention and Economic Growth1966 (1966).

7. Damanpour, Fariborz, and William M. Evan. "Organizational innovation and performance: the problem of" organizational lag"." Administrative science quarterly (1984): 392-409.

8. Park, Eun-Mi, and Joung-Hae Seo. "Effects of exploration and exploitation activities on patent capacity and innovation performance: Moderating effects of absorptive capacity." Indian Journal of Public Health Research \& Development 9, no. 9 (2018): 1295-1302.

9. Park, Eun Mi, and Seong Taek Park. "The effectiveness of absorptive capacity formation mechanism on innovation performance by industry." Indian Journal of Science and Technology 8, no. 21 (2015): 19.

10. Cohen, Wesley M., and Daniel A. Levinthal. "Absorptive capacity: A new perspective on learning and innovation." Administrative science quarterly (1990): 128-152.

11. Lynn, Gary S., Richard R. Reilly, and Ali E. Akgun. "Knowledge management in new product teams: practices and outcomes." IEEE transactions on Engineering Management 47, no. 2 (2000): 221-231.

12. Madhavan, Ravindranath, and Rajiv Grover. "From embedded knowledge to embodied knowledge: New product development as knowledge management." Journal of marketing 62, no. 4 (1998): 1-12.

13. Lane, Peter J., and Michael Lubatkin. "Relative absorptive capacity and interorganizational learning." Strategic management journal 19, no. 5 (1998): 461-477.

14. Zahra, Shaker A., and Gerard George. "The net-enabled business innovation cycle and the evolution of dynamic capabilities." Information systems research 13, no. 2 (2002): 147-150.

15. Park, Seong-Taek, and Young-Ki Kim. "Artificial Intelligence Research Trend Using SCOPUS DB: 2011-2019. " International Journal of Advanced Science and Technology 29, no. 4s (2020): 304-312.

16. Diesner, Jana, Kathleen M. Carley, and Laurent Tambayong. "Extracting socio-cultural networks of the Sudan from open-source, large-scale text data." Computational and Mathematical Organization Theory 18, no. 3 (2012): 328-339.

17. Watts, Duncan J. "Networks, dynamics, and the small-world phenomenon." American Journal of sociology 105, no. 2 (1999): 493-527.

18. Blei, David M., Andrew Y. Ng, and Michael I. Jordan. "Latent dirichlet allocation." In Advances in neural information processing systems, pp. 601-608. 2002.

19. Blei, David M., Andrew Y. Ng, and Michael I. Jordan. "Latent dirichlet allocation." Journal of machine Learning research 3, no. Jan (2003): 993-1022.

20. Ramos, Juan. "Using tf-idf to determine word relevance in document queries." In Proceedings of the first instructional conference on machine learning, vol. 242, pp. 133-142. 2003. 